\title{
STABILITY CONDITIONS OF A CLASS OF LINEAR RETARDED DIFFERENTIAL SYSTEMS
}

\author{
SERBUN UFUK DEĞER ${ }^{1, *}$ AND YAŞAR BOLAT ${ }^{2}$ \\ ${ }^{1}$ Institute of Sciences, Kastamonu University, Kastamonu, TURKEY \\ ${ }^{2}$ Department of Mathematics, Faculty of Art $\&$ Science, Kastamonu University, Kastamonu, TURKEY
}

*Corresponding author: sudeger@kastamonu.edu.tr

AbStRaCt. In this paper, we give some new necessary and sufficient conditions for the asymptotic stability of a linear retarded differential system with two delays

$$
x^{\prime}(t)+(1-a) x(t)+A(x(t-k)+x(t-l))=0, \quad t \geq 0,
$$

where $a<1$ is a real number, $A$ is a $2 \times 2$ real constant matrix, and $k, l$ are positive numbers such that $k>l$.

\section{INTRODUCTION AND PRELIMINARIES}

Retarded differential equations are a type of differential equation in which the derivative of the unknown function at a certain time is given in terms of the values of the function at previous times. Stability of these equation has a wide range of applications in science and engineering. Recently, These equations have been investigated by many authors; for example, Matsunaga [1], Cooke and van den Driessche [2], Kuang [3] , Cooke and Grossman [4], Ruan and Wei [5], Hale and Lunel [6], Khokhlovaa, Kipnis and Malygina [8], Cermák and Jánsky [9], Hrabalova [10], Nakajima [11], Hara and Sakata [12], Smith [13], Freedman and Kuang [14] and Bellman and Cooke [15] which have studied the asymptotic stability of linear retarded

Received 2018-03-09; accepted 2018-05-09; published 2018-07-02.

2010 Mathematics Subject Classification. 39A13, 39A30.

Key words and phrases. differential equations; characteristic equation; asymptotic stability.

(C)2018 Authors retain the copyrights of their papers, and all open access articles are distributed under the terms of the Creative Commons Attribution License. 
differential equations. In this paper, we give some new necessary and sufficient conditions for the asymptotic stability of the following system

$$
x^{\prime}(t)+(1-a) x(t)+A(x(t-k)+x(t-l))=0, \quad t \geq 0,
$$

where $a<1$ is a real number, $A$ is a $2 \times 2$ real constant matrix, and $k, l$ are positive numbers such that $k>l$.

System (1.1) is called a retarded or delay differential system if the highest derivative term does not have a delay.The characteristic equations of retarded differential equations are polynomials. These polynomials are exponential polynomials or quasi-polynomials as named in Bellman and Cooke [15]. We know that for the linear retarded differential equation, the zero solution being asymptotically stable is equivalent to all solutions having limit zero as $t \rightarrow \infty$ which in turn is true if and only if all roots of the associated characteristic equation have negative real parts.

The purpose of this paper is to obtain new results for the asymptotic stability of zero solution of system (1.1) when $A$ is a constant matrix. Now we will give some basic information that we use for the lemmas. If we get $x(t)=P y(t)$ for a regular matrix $P$ in (1.1), then we obtain the following system;

$$
y^{\prime}(t)+(1-a) y(t)+P^{-1} A P(y(t-k)+y(t-l))=0, \quad t \geq 0
$$

Thus, matrix $A$ can be given in one of the following two matrices in Jordan form[7]:

$$
\begin{aligned}
& \text { (I) } A=\left(\begin{array}{cc}
q_{1} & p \\
0 & q_{2}
\end{array}\right), b_{1}, b_{2} \text { and } p \text { are real constants, } \\
& \text { (II) } A=q\left(\begin{array}{cc}
\cos \theta & -\sin \theta \\
\sin \theta & \cos \theta
\end{array}\right), \quad q, \theta \text { are real constants and }|\theta|<\frac{\pi}{2} .
\end{aligned}
$$

Here we discuss the case $(I I)$, the other case should be discussed similarly. The characteristic equation of system (1.1) is given as

$$
F(\lambda):=\operatorname{det}\left(\lambda I_{2}+(1-a) I_{2}+A\left(e^{-\lambda k}+e^{-\lambda l}\right)\right)=0
$$

where $I_{2}$ is the $2 \times 2$ identity matrix. By the case $(I I)$, we have $F(\lambda)$ as follows

$$
F(\lambda) \equiv f_{\theta}(\lambda) \overline{f_{\theta}(\bar{\lambda})}=0
$$

where

$$
f_{\theta}(\lambda)=\left(\lambda+(1-a)+q\left(e^{-\lambda k+i|\theta|}+e^{-\lambda l+i|\theta|}\right)\right)
$$

and $\bar{\lambda}$ is the complex conjugate of any complex $\lambda$. Note that $\overline{f_{\theta}(\bar{\lambda})}=0$ implies $f_{\theta}(\bar{\lambda})=0$. 


\section{Some Auxiliary Lemmas}

Lemma 2.1. The zero solution of (1.1) is asymptotically stable if and only if all the roots of equation

$$
f_{\theta}(\lambda, q)=\lambda+(1-a)+q\left(e^{-\lambda k+i|\theta|}+e^{-\lambda l+i|\theta|}\right)
$$

lie in the left half of the complex plane.

Since $f_{\theta}$ is an analytic function of $\lambda$ and $q$ for the fixed numbers $k, l, a$ and $\theta$, one can regard the root $\lambda=\lambda(q)$ of (2.1) as a continuous function of $q$. The next lemma plays very important role for the main theorem.

Lemma 2.2. As q varies, the sum of the multiplicities of the roots of (2.1) in the open right half-plane can change only if a root appears on or crosses the imaginary axis.

Consequently, we claim that (2.1) has only imaginary roots $\pm i \omega$. We will determine the value of $q$ as equation (2.1) has roots on the imaginary axis. Now, we can write the characteristic equation (2.1) as follows;

$$
\lambda+(1-a)+q\left(e^{-\lambda k+i \theta}+e^{-\lambda l+i \theta}\right)=0 .
$$

Let $\lambda=i \omega$ is a root $(2.2)$ such that $\omega \in \mathbb{R}$. Firsty, since $f_{\theta}(0) \neq 0$, we see that $\omega \neq 0$. If $\omega \neq 0$, then we write

$$
i \omega+(1-a)+q\left(e^{-i \omega k+i \theta}+e^{-i \omega l+i \theta}\right)=0,
$$

and from this equation, we have

$$
\left\{\begin{array}{c}
\omega=q(\sin (\omega k-\theta)+\sin (\omega l-\theta)) \\
a-1=q(\cos (\omega k-\theta)+\cos (\omega l-\theta)),
\end{array}\right.
$$

which is equivalent to

$$
\left\{\begin{array}{c}
\omega=2 q \sin \left(\frac{\omega(k+l)}{2}-\theta\right) \cos \left(\frac{\omega(k-l)}{2}\right) \\
a-1=2 q \cos \left(\frac{\omega(k+l)}{2}-\theta\right) \cos \left(\frac{\omega(k-l)}{2}\right) .
\end{array}\right.
$$

From (2.4), we get

$$
\frac{\omega}{a-1}=\tan \left(\frac{\omega(k+l)}{2}-\theta\right)
$$

We know that the function tanjant is defined as on the region

$$
H=\left\{(t, \tan t): t \in \mathbb{R}, t=\frac{\pi}{2}+\rho \pi, \rho \in \mathbb{Z}\right\} .
$$

Thus, (2.5) has only a sequence of the roots $\left\{\omega_{j}: j \geq 1\right\}$, where

$$
\omega_{j} \in\left(\frac{(2 j-1) \pi+2 \theta}{k+l}, \frac{(2 j+1) \pi+2 \theta}{k+l}\right) \quad \text { for } \omega_{j}>0
$$

and

$$
\omega_{j} \in\left(\frac{-(2 j+1) \pi+2 \theta}{k+l}, \frac{-(2 j-1) \pi+2 \theta}{k+l}\right) \quad \text { for } \omega_{j}<0
$$


Lemma 2.3. Suppose that $\left\{q_{j}: j \geq 1\right\}>0$ and $0<\theta<\frac{\pi}{2}$. Let $\lambda=i \omega_{j}$ be a root of (2.1) where $\omega_{j} \in$ $\left(\frac{(3-4 j) \pi}{k-l}, \frac{(4 j-3) \pi}{k-l}\right)-\left\{\frac{-n \pi+2 \theta}{k+l}, \frac{n \pi+2 \theta}{k+l}\right\}$ is a real number for $n \in \mathbb{N}$. Then the following conditions hold:

(i) If $\left(2 q_{j} \cos \left(\frac{\omega_{j}(k-l)}{2}\right)\right)^{2}-(a-1)^{2} \leq 0$, then there exists no real number $\omega_{j}$.

(ii) If $\left(2 q_{j} \cos \left(\frac{\omega_{j}(k-l)}{2}\right)\right)^{2}-(a-1)^{2}>0$, then there exist the real numbers $\omega_{j}$ and $q_{j}, q_{j}$ is as follows: $q_{j}=\frac{a-1}{2 \cos \left(\frac{\omega_{j}(k+l)}{2}-\theta\right) \cos \left(\frac{\omega_{j}(k-l)}{2}\right)}$.

Remark 2.1. In case $\left(2 q_{j} \cos \left(\frac{\omega_{j}(k-l)}{2}\right)\right)^{2}-(a-1)^{2}>0$, for the equality

$$
\omega_{j}= \pm \varphi_{j}= \pm \sqrt{\left(2 q_{j} \cos \left(\frac{\omega_{j}(k-l)}{2}\right)\right)^{2}-(a-1)^{2}}
$$

the sum of delays $k$ and $l$ is as follows;

$$
\begin{gathered}
\left(k_{n}+l_{n}\right)^{+}=\frac{2}{\varphi_{j}}\left\{-(2 n+2) \pi+\arccos \left(\frac{a-1}{2 q_{j} \cos \left(\frac{\omega_{j}(k-l)}{2}\right)}\right)+\theta\right\} \\
\left(k_{n}+l_{n}\right)^{-}=\frac{2}{\varphi_{j}}\left\{-2 n \pi+\arccos \left(\frac{a-1}{2 q_{j} \cos \left(\frac{\omega_{j}(k-l)}{2}\right)}\right)-\theta\right\},
\end{gathered}
$$

for $n \in \mathbb{N}$. Also, $i \varphi_{j}$ or $-i \varphi_{j}$ is a root of $(2.1)$ for the sum of delays $\left(k_{n}+l_{n}\right)^{+}$or $\left(k_{n}+l_{n}\right)^{-}$for $n \in \mathbb{N}$.

Proof of Lemma 2.3. By (2.4), we can write

$$
\omega^{2}+(a-1)^{2}=2 q \cos \left(\frac{\omega(k-l)}{2}\right)
$$

Substituting $\omega=\left\{\omega_{j}\right\}_{j \geq 1}$ and $q=\left\{q_{j}\right\}_{j \geq 1}$ into the above equation, we obtain

$$
\omega_{j}^{2}+(a-1)^{2}=2 q_{j} \cos \left(\frac{\omega_{j}(k-l)}{2}\right)
$$

If $\left(2 q_{j} \cos \left(\frac{\omega_{j}(k-l)}{2}\right)\right)^{2}-(a-1)^{2} \leq 0$, then statement (2.6) implies $\omega_{j}^{2}<0$, contradicts $\omega_{j}^{2}>0$; thus, condition $(i)$ is verified; that is, (2.1) has no root on the imaginary axis for all $k>l>0$.

On the other hand, if $\left(2 q_{j} \cos \left(\frac{\omega_{j}(k-l)}{2}\right)\right)^{2}-(a-1)^{2}>0$, statement $(2.6)$ implies $\omega_{j}= \pm \varphi_{j}$ for $\varphi_{j}=$ $\sqrt{\left(2 q_{j} \cos \left(\frac{\omega_{j}(k-l)}{2}\right)\right)^{2}-(a-1)^{2}}$. From (2.4), we get

$$
q_{j}=\frac{a-1}{2 \cos \left(\frac{\omega_{j}(k+l)}{2}-\theta\right) \cos \left(\frac{\omega_{j}(k-l)}{2}\right)} .
$$

Now we will show that $i \varphi_{j}$ is a root of $(2.1)$. In case

$$
\omega_{j}=\left(2 q_{j} \cos \left(\frac{\omega_{j}(k-l)}{2}\right)\right)^{2}-(a-1)^{2},
$$


(2.4) and $\cos \left(\frac{\omega_{j}(k-l)}{2}\right)>0$ implies $\sin \left(\frac{\omega_{j}(k+l)}{2}-\theta\right)>0$. Thus, we can write

$$
\frac{\omega_{j}(k+l)}{2}-\theta=-(2 n+2) \pi+\arccos \left(\frac{a-1}{2 q_{j} \cos \left(\frac{\omega_{j}(k-l)}{2}\right)}\right) \text { for } n \in \mathbb{N}
$$

which yields $\left(k_{n}+l_{n}\right)^{+}$. After that, we have

$$
\sin \left(\arccos \left(\frac{a-1}{2 q_{j} \cos \left(\frac{\omega_{j}(k-l)}{2}\right)}\right)\right)=\frac{\varphi_{j}}{2 q_{j} \cos \left(\frac{\omega_{j}(k-l)}{2}\right)}
$$

because of

$$
\arccos \left(\frac{a-1}{2 q_{j} \cos \left(\frac{\omega_{j}(k-l)}{2}\right)}\right)= \begin{cases}\arcsin \left(\frac{\varphi_{j}}{2 q_{j} \cos \left(\frac{\omega_{j}(k-l)}{2}\right)}\right) & \text { if } a-1>0 \\ \pi-\arcsin \left(\frac{\varphi_{j}}{2 q_{j} \cos \left(\frac{\omega_{j}(k-l)}{2}\right)}\right) & \text { if } a-1<0 .\end{cases}
$$

For the case $k+l=\left(k_{n}+l_{n}\right)^{+}$, from $(2.1)$ we have

$$
\begin{aligned}
f(i \omega) & =i \omega+(1-a)+q\left(e^{i(\omega k-\theta)}+e^{i(\omega l-\theta)}\right) \\
& =i \sqrt{\left(2 q_{j} \cos \left(\frac{\omega_{j}(k-l)}{2}\right)\right)^{2}-(a-1)^{2}}+(1-a)+ \\
& +2 q_{j} \cos \left(\frac{\omega_{j}(k-l)}{2}\right) e^{-i\left(\frac{\omega_{j}(k+l)}{2}-\theta\right)} \\
& =i \sqrt{\left(2 q_{j} \cos \left(\frac{\omega_{j}(k-l)}{2}\right)\right)^{2}-(a-1)^{2}}+(1-a)+ \\
& +2 q_{j} \cos \left(\frac{\omega_{j}(k-l)}{2}\right) e^{-i}\left(-(2 n+2) \pi+\arccos \left(\frac{a-1}{2 q_{j} \cos \left(\frac{\omega_{j}(k-l)}{2}\right)}\right)\right) \\
& =i \sqrt{\left(2 q_{j} \cos \left(\frac{\omega_{j}(k-l)}{2}\right)\right)^{2}-(a-1)^{2}}+(1-a)+ \\
& +2 q_{j} \cos \left(\frac{\omega_{j}(k-l)}{2}\right)\left\{\cos \left(\arccos \left(\frac{a-1}{2 q_{j} \cos \left(\frac{\omega_{j}(k-l)}{2}\right)}\right)\right)\right\}- \\
& -2 q_{j} \cos \left(\frac{\omega_{j}(k-l)}{2}\right)\left\{i \sin \left(\arccos \left(\frac{a-1}{2 q_{j} \cos \left(\frac{\omega_{j}(k-l)}{2}\right)}\right)\right)\right\} \\
& =i \sqrt{\left(2 q_{j} \cos \left(\frac{\omega_{j}(k-l)}{2}\right)\right)^{2}-(a-1)^{2}+(1-a)+} \\
& +(1-a)-i \sqrt{\left(2 q_{j} \cos \left(\frac{\omega_{j}(k-l)}{2}\right)\right)^{2}-(a-1)^{2}} \\
& =0 ;
\end{aligned}
$$

thus, we can see that $i \varphi_{j}$ is a root of $(2.1)$. Similarly, when $\omega_{j} \in\left(\frac{(3-4 j) \pi}{k-l}, 0\right],-i \varphi_{j}$ is a root of (2.1) for the sum of delays $\left(k_{n}+l_{n}\right)^{-}$. The proof is completed. 
When $\omega_{j}<0$, we have the following analogous result.

Lemma 2.4. Suppose that $\left\{q_{j}: j \geq 1\right\}<0$ and $0<\theta<\frac{\pi}{2}$. Let $\lambda=i \omega_{j}$ be a root of (2.1) where $\omega_{j} \in$ $\left(\frac{(4 j-3) \pi}{k-l}, \frac{(4 j-1) \pi}{k-l}\right) \cup\left(\frac{(1-4 j) \pi}{k-l}, \frac{(3-4 j) \pi}{k-l}\right)-\left\{\frac{-n \pi+2 \theta}{k+l}, \frac{n \pi+2 \theta}{k+l}\right\}$ for $n \in \mathbb{N}$. Then the following conditions hold:

(i) If $\left(2 q_{j} \cos \left(\frac{\omega_{j}(k-l)}{2}\right)\right)^{2}-(a-1)^{2} \leq 0$, then there exists no real number $\omega_{j}$.

(ii) If $\left(2 q_{j} \cos \left(\frac{\omega_{j}(k-l)}{2}\right)\right)^{2}-(a-1)^{2}>0$, then there exist the real numbers $\omega_{j}$ and $q_{j}$, $q_{j}$ is as follows: $q_{j}=\frac{a-1}{2 \cos \left(\frac{\omega_{j}(k+l)}{2}-\theta\right) \cos \left(\frac{\omega_{j}(k-l)}{2}\right)}$.

Proof. The proof is similar of the lemma 2.3 .

Lemma 2.5. Suppose that $a<1$. Let $\lambda(q)=\operatorname{Re}(\lambda(q))+i \operatorname{Im}(\lambda(q))$ the root of $(2.1)$ satisfying $\operatorname{Re}\left(\lambda\left(q_{j}\right)\right)=$ $0, \operatorname{Im}\left(\lambda\left(q_{j}\right)\right)=\omega_{j}$. Then the following equation is provided:

$$
\text { Si } g n \operatorname{Re}\left(\lambda^{\prime}\left(q_{j}\right)\right) \text {. Si gn } q_{j}>0
$$

Proof. Taking the derivative of $\lambda$ with respect to $q$ on $(2.1)$, we have

$$
\begin{aligned}
\frac{d \lambda}{d q}+\left(e^{-\lambda k+i \theta}\right. & \left.+e^{-\lambda l+i \theta}\right)+q\left(-k e^{-\lambda k+i \theta}-l e^{-\lambda l+i \theta}\right) \frac{d \lambda}{d q}=0 \\
\frac{d \lambda}{d q} & =-\frac{e^{-\lambda k+i \theta}+e^{-\lambda l+i \theta}}{1-q\left(k e^{-\lambda k+i \theta}+l e^{-\lambda l+i \theta}\right)} \\
& =\frac{\lambda+1-a}{q\left(1-q\left(k e^{-\lambda k+i \theta}+l e^{-\lambda l+i \theta}\right)\right)}
\end{aligned}
$$

Substituting $\omega=\omega_{j}$ and $q=q_{j}$ into the above equation, we get

$$
\left.\frac{d \lambda}{d q}\right|_{\lambda=i \omega}=\frac{i \omega_{j}+1-a}{q_{j}\left(1-q_{j}\left(k e^{-i\left(\omega_{j} k-\theta\right)}+l e^{-i\left(\omega_{j} l-\theta\right)}\right)\right)}
$$

Thus, it follows that;

$$
\begin{aligned}
\left.\operatorname{Re} \frac{d \lambda}{d q}\right|_{\lambda=i \omega} & =\frac{(1-a) q_{j}\left(1-q_{j}\left(k \cos \left(\omega_{j} k-\theta\right)+l \cos \left(\omega_{j} l-\theta\right)\right)\right)}{M}+ \\
& +\frac{\omega_{j} q_{j}^{2}\left(k \sin \left(\omega_{j} k-\theta\right)+l \sin \left(\omega_{j} l-\theta\right)\right)}{M}
\end{aligned}
$$

where

$$
M=q_{j}^{2}\left(1-q_{j}\left(k \cos \left(\omega_{j} k-\theta\right)+l \cos \left(\omega_{j} l-\theta\right)\right)\right)^{2}+q_{j}^{4}\left(k \sin \left(\omega_{j} k-\theta\right)+l \sin \left(\omega_{j} l-\theta\right)\right)^{2} .
$$

Let $A_{1}=\sin (\omega k-\theta)+\sin (\omega l-\theta)$ and $A_{2}=\cos (\omega k-\theta)+\cos (\omega l-\theta)$.

By $(2.4)$, we have $\frac{A_{1}(\omega)}{A_{2}(\omega)}=\tan \left(\frac{\omega(k+l)}{2}-\theta\right)$, thus

$$
\frac{d}{d \omega}\left(\frac{A_{1}(\omega)}{A_{2}(\omega)}\right)=\frac{A_{1}^{\prime}(\omega) A_{2}(\omega)-A_{2}^{\prime}(\omega) A_{1}(\omega)}{\left(A_{2}(\omega)\right)^{2}}>0
$$

is obtained, which implies $A_{1}^{\prime}(\omega) A_{2}(\omega)-A_{2}^{\prime}(\omega) A_{1}(\omega)>0$. 
Since $A_{1}^{\prime}=k \cos (\omega k-\theta)+l \cos (\omega l-\theta)$ and $A_{2}^{\prime}=-k \sin (\omega k-\theta)-l \sin (\omega l-\theta),(2.9)$ can written

$$
\operatorname{Re} \frac{d \lambda}{d q} \underset{\lambda=i \omega}{\mid}=\frac{q_{j}\left[\left((1-a)-(1-a) q_{j} A_{1}^{\prime}(\omega)\right)-\omega_{j} q_{j} A_{2}^{\prime}(\omega)\right]}{M}
$$

we use (2.4) for above equation, then we get

$$
\operatorname{Re} \frac{d \lambda}{d q} \underset{\lambda=i \omega}{\mid}=\frac{q_{j}\left[(1-a)+q_{j}^{2}\left(A_{1}^{\prime}(\omega) A_{2}(\omega)-A_{2}^{\prime}(\omega) A_{1}(\omega)\right)\right]}{M} .
$$

Hence, the proof is completed.

\section{Main Results}

Theorem 3.1. Suppose that $a<1,0<\theta<\frac{\pi}{2}$ and the matrix $A$ of the system (1.1) is written as the form $(I I)$. we define

$$
q_{\theta}^{-}=\max _{j \geq 1}\left\{q_{j}: q_{j}<0\right\}, \quad q_{\theta}^{+}=\min _{j \geq 1}\left\{q_{j}: q_{j}>0\right\},
$$

and let a neighborhood of $q=0$ is $\left(q_{\theta}^{-}, q_{\theta}^{+}\right)$. Then system (1.1) is asymptotically stable if and only if

$$
q_{\theta}^{-}<q<q_{\theta}^{+}
$$

Proof. In case of $q=0$, the root of (2.1) is only $\lambda(0)=a-1<0$. Thus, the root of the equation (2.1) has a negative real part. By the continuity of the roots with respect to $q$ and by the asymptotic stability of (1.1), we can claim that the roots of equation $(2.1)$ are inside a neighborhood $\left(q_{\theta}^{-}, q_{\theta}^{+}\right)$of $q=0$.

Since $\lambda(0)<0$, in case of $q_{\theta}^{-}<\infty$, by Lemma 2.4, equation (2.1) has roots on the imaginary axis because of $q_{\theta}^{-}$is the first value $q<0$. By Lemma 2.2, all roots of the equation (2.1) have negative real parts for $\left(q_{\theta}^{-}, 0\right]$. Similarly, in case of $q_{\theta}^{+}<\infty$, by Lemma 2.3, equation (2.1) has roots on the imaginary axis because of $q_{\theta}^{+}$is the first value $q>0$. By Lemma 2.2, all roots of the equation (2.1) have negative real parts for $\left[0, q_{\theta}^{+}\right)$. Also, by Lemma 2.5, equation (2.1) has at least one root positive real part for $\left(-\infty, q_{\theta}^{-}\right)$and $\left(q_{\theta}^{+}, \infty\right)$. Thus, the proof is completed.

Theorem 3.2. Suppose that $a<1, \theta=0$ and the matrix $A$ of the system (1.1) is written as the form (II). we define

$$
q_{0}^{-}=\max _{j \geq 1}\left\{\frac{a-1}{2}: a<1\right\}, \quad q_{0}^{+}=\min _{j \geq 1}\left\{q_{j}: q_{j}>0\right\},
$$

and let a neighborhood of $q=0$ is $\left(\frac{a-1}{2}, q_{0}^{+}\right)$. Then system (1.1) is asymptotically stable if and only if

$$
\frac{a-1}{2}<q<q_{0}^{+}
$$

Proof. When $\theta=0$, equation (2.1) has only root $\lambda=0$ as $q=\frac{a-1}{2}$, that is $\lambda\left(\frac{a-1}{2}\right)=0$. Since

$$
\frac{d \lambda\left(\frac{a-1}{2}\right)}{d q}<0
$$


we can write $q_{0}^{-}=\max _{j \geq 1}\left\{q_{j}=\frac{a-1}{2}: a<1, j \geq 1\right\}$. Since the rest of the proof is similar to the Theorem 3.11 , it is obvious.

Theorem 3.3. Suppose that $a<1$, and the matrix $A$ of the system (1.1) is written as the form $(I)$. we define

$$
q^{-}=\max _{j \geq 1}\left\{q_{j}: q_{j}<0\right\}, \quad q^{+}=\min _{j \geq 1}\left\{q_{j}: q_{j}>0\right\},
$$

and let a neighborhood of $q=0$ is $\left(q^{-}, q^{+}\right)$. Then system (1.1) is asymptotically stable if and only if

$$
q^{-}<q_{1}, q_{2}<q^{+}
$$

Proof. The proof is similar to the Theorem 3.1.

\section{REFERENCES}

[1] H. Matsunaga, Delay Dependent and Delay Independent Stability Criteria For A Delay Differential System, American Mathematical Society, 136 Fields Inst. Commun. 42 (2008), 4305-4312.

[2] K. L. Cooke and P. van den Driessche, On zeroes of some transcendental equations, Funkcial.Ekvacioj, 29 (1986), 77-90.

[3] Y. Kuang, Delay Differential Equations with Applications in Population Dynamics, Academic Press, Boston, 1993.

[4] K. L. Cooke and Z. Grossman, Discrete delay, distributed delay and stability switches, J. Math. Anal. Appl. 86 (1982), 592-627.

[5] S.Ruan and J.Wei, On The Zeros Of Transcendental Functions With Applications To Stability Of Delay Differential Equations With Two Delays, Dynamic of Continuous, Discr. impuls. Syst. (2003), 863-874.

[6] J.K.Hale and S. M. Verduyn Lunel, Introduction to Functional Differential Equations, Springer-Verlag, New York, 1993.

[7] S. Elaydi, An Introduction to Difference Equations, 3rd ed., Springer-Verlag, New York, 2005.

[8] T. Khokhlovaa, M. Kipnis, V. Malygina, Discrete delay, The stability cone for a delay differential matrix equation, Appl. Math. Lett. 24 (2011), 742-745.

[9] J. Cermák, J.Jánsky, Stability switches in linear delay difference equations, Appl. Math. Comput. 243 (2014) $755-766$.

[10] Jana Hrabalova, Stability Properties of a Discrrtized Neutral Delay Differential Equation, Tatra Mt. Math. Publ. 54 (2013), 83-92

[11] H. Nakajima, On the Stability of a linear Retarded Differential-Difference Equation, Funkcialaj Ekvacioj. 57 (2014), 43-56

[12] T. Hara, S. Sakata, An application of the Hurwitz theorem to the root analysis of the characteristic equation, Appl. Math. Lett. 24 (2011) $12-15$

[13] H. Smith, An Introduction to Delay Differential Equations with Applications to The Life Science, Springer, New York 2010.

[14] H. I .Freedman, Y.Kuang, Stability switches in linear scalar neutral delay equation, Funkcial. Ekvac. 34 (1991), $187-209$.

[15] R. Bellman and K. L. Cooke, Differential-Difference Equations, Academic Press, New York, 1963. 